\title{
One Pot Synthesis of Some Novel Sulfonamide Derivatives Containing -NH2 Group: Spectral Characterization and Biological Evaluation
}

Hajira Rehman ${ }^{1 *}$, Abdul Qadir M1, Hazoor Ahmad Shad ${ }^{2,3^{*}}$, and Zafar lqbal Khan ${ }^{4}$

${ }^{1}$ Institute of Chemistry, University of the Punjab, Lahore, Pakistan

${ }^{2}$ Department of Chemistry, University of Sargodha, Pakistan

${ }^{3}$ Department of Chemistry, Postgraduate College Jhang, Pakistan

${ }^{4}$ Department of Botany, University of Sargodha, Sargodha, Pakistan

\begin{abstract}
A series of sulfonamide derivatives HR1-HR5 were synthesized in one step reaction (nucleophilic substitution reaction $\mathrm{SN}^{2}$ ). Structures of new products were confirmed by elemental and spectral analysis i.e., FTIR, UV, ${ }^{1} \mathrm{H}$ NMR, ${ }^{13} \mathrm{C}$ NMR, EIS-MS. In-vitro, antibacterial and anti-fungal activity of newly synthesized compounds was investigated against two bacterial strains: Escherichia coli and Staphylococcus aureus and two fungal strains: Aspergillum flavous and Aspergillum niger. It was found that among all tested compounds HR2 showed good antibacterial activity with MIC $1.13 \times 10^{-3}$ and $1.54 \times 10^{-3}$ for $S$. aureus and E. coli respectively. While HR4 showed good antifungal activity with inhibition zone $25.2 \pm 0.12 \mathrm{~mm}$ (MIC: $71.2 \times 10^{-3} \mathrm{~mol} / \mathrm{L}$ ) and $17.1 \pm 55.5 \mathrm{~mm}$ (MIC: 98.9 $\times 10^{-3} \mathrm{~mol} / \mathrm{L}$ ) against $A$. flavous and $A$. niger respectively. Developed compounds were also screened for their $\mathrm{In}$ vitro antioxidant activity by DPPH radical scavenging assay. All compounds showed moderate activity but potential activity with $15.75 \%$ at $6 \mathrm{mM}$ was exhibited by compound HR2.
\end{abstract}

Keywords: Antibacterial activity; Antifungal activity; p-Toluene sulfonamides; Sulfonamide

\section{Introduction}

Sulfonamides have commercialized applications as antibacterial antibiotic agents as they inhibit activity of enzyme dihydropteroate synthase (DHPS) [1] and prevent synthesis of folic acid (Vitamin B9), which is an essential intermediate for life of bacteria. So sulfonamides and their derivatives are used as antibiotics medicines [2]. Apart from this application as an antibacterial agent, various sulfonamide derivatives are known to inhibit many enzymes such as Serine protease [3-5], cyclooxygenase [6], matrix metalloproteinase [7] and carbonic anhydrase [8-11]. Moreover their widespread potential values have led to discovery of various therapeutic applications in cancer chemotherapy, hypoglycemia, diuretics [12] and anti-impotence agent Viagra [13]. They have also received a considerable attention due to their diverse biological activities as HIV protease [14,15] and as an antitumor [16]. In recent year, novel sulfonamides were synthesized such as doriperiens with brand name doribax which is an injectable antibiotic [17]. Other developed sulfonamides drugs such as AZA (acetazolamide) and MZA (metahacetazoamides) are widely used mainly as anti-glucoma agents and also used for therapy of some other diseases [18-20]. Ester derivatives of sulfonamides are well known as cell proliferation inhibitor [21]. Newer sulfonamides and their derivatives have also got more attraction in the field of medicine $[22,23]$. On other hand disease causing organisms when treated with routine antibiotic become must resistance with appearance of some additional species as per mutation. So synthesis of novel sulfonamides and their derivatives have got much attention from researcher because of their widespread applications in the field of medical chemistry and medicine science [24,25]. Most practical and different method for the synthesis of sulfonamides (Scheme 1) involves sulfonation of amines and alcohols [26] in presence of some base such as Pyridine, triethylamine, or some metal hydroxides or carbonates.

In present study, we have synthesized five new sulfonamides (HR1-HR5) (Figure 1) derivatives by reaction of amine $\left(-\mathrm{NH}_{2}\right.$ group containing drug) and p-toluene sulfonyl chloride. These $-\mathrm{NH}_{2}$ group containing drugs are cefotexime, ciprofloxacin, frusamide, amlodepine and indapamide respectively. These synthesized compounds not yet reported which is a strong evidence of their novelty. Synthesized compounds were biologically evaluated by using the bacterial and two fungal strains such as Escherichia coli, Staphylococcus aureus, Aspergillum niger and Aspergillum flavous.

\section{Experimental}

\section{Chemistry}

Chemicals used in present work were of analytical grade and used without further purification to synthesize desired compounds. Chemicals were obtained from E-Merck (Germany) and BDH (UK). Grade 1 quality water $(0.01 \mu \mathrm{S} / \mathrm{cm})$ was prepared in our own laboratory. ${ }^{1} \mathrm{H}$ NMR spectra were developed on Bruker spectrometer $400 \mathrm{MHz}$.

Finnigan MAT 112 mass spectrometer was used for recording MS data. Elementary analysis of compound was conducted by using Elmer elemental analyzer. Gallen kamp MP70 was used to determine the m.p. For recording infrared spectra, Cary 630 Agilent FFIR was used. Absorption spectra were conducted on PGT90+UV-VIS spectrometer. Pre-coated TLC silica plates (Merck, Germany) were used for purification and to confirm progress of the synthesized compounds.

\section{General procedure for synthesis of compounds}

For the preparation of sulfonamide derivatives (HR1-HR5), a proficient method based on Hinsberg Test was used i.e., sulfonylation of primary, secondary amine in presence of a base resulting in nucleophilic attack by amine. Tosylchloride was used for sulfonylation and $\mathrm{Na}_{2} \mathrm{CO}_{3}$

*Corresponding author: Hazoor Ahmad Shad, Department of Chemistry, University of Sargodha, Pakistan, Tel: +923323177994; E-mail: hazoorahmad@gmail.com

Received September 15, 2016; Accepted September 26, 2017; Published September 29, 2017

Citation: Rehman H, Abdul Qadir M, Shad HA, Khan ZI (2017) One Pot Synthesis of Some Novel Sulfonamide Derivatives Containing -NH2 Group: Spectral Characterization and Biological Evaluation. Med Chem (Los Angeles) 7: 252-256. doi: 10.4172/2161-0444.1000465

Copyright: @ 2017 Rehman H, et al. This is an open-access article distributed under the terms of the Creative Commons Attribution License, which permits unrestricted use, distribution, and reproduction in any medium, provided the original author and source are credited. 


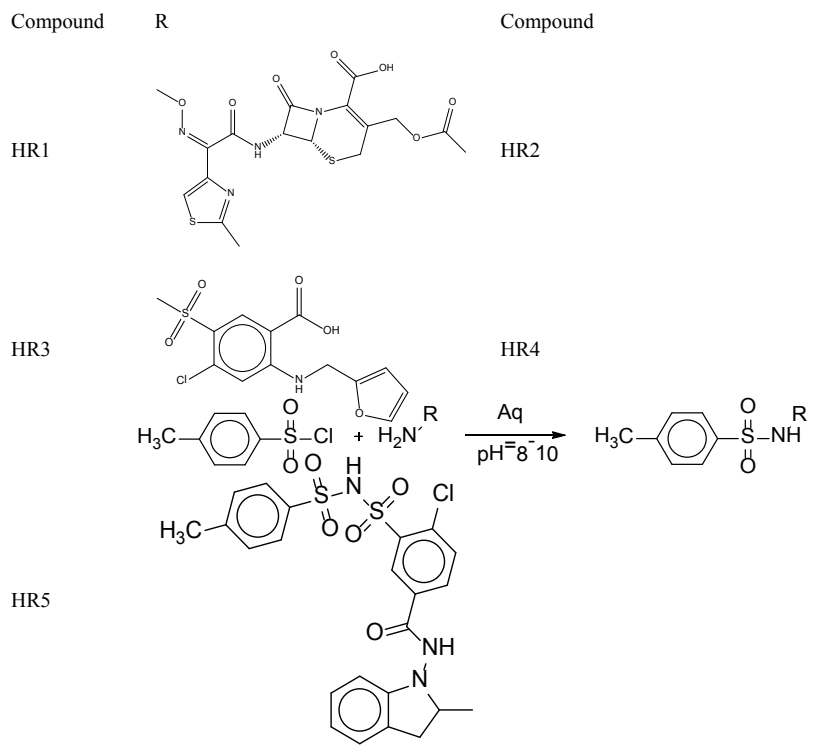

Figure 1: New sulphonamides (HR1-HR5).

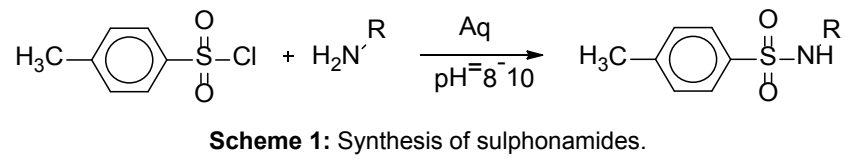

as base was used for neutralization of generated $\mathrm{HCl}$. It is a one pot reaction. Drug containing $-\mathrm{NH}_{2}$ group $(0.001 \mathrm{M})$ was stirred in water while maintain the $\mathrm{pH}$ at 9 , then sulfonyl chloride $(0.001 \mathrm{M})$ was added and mixture was stirred $2 \mathrm{~h}$. Precipitates were filtered and purified by using preparatory TLC.

\section{Antimicrobial activity}

Luria-Bertain broth was used as Growth media as it is highly supportive in bacterial growth [27]. In order to make media, $4.0 \mathrm{~g}$ of Tryptone, $2.0 \mathrm{~g}$ of yeast extract and $4.0 \mathrm{~g}$ of sodium chloride were added in $400 \mathrm{~mL}$ distilled water. The $p \mathrm{H}$ value of media was kept at 7. The media was then sterilized in autoclave at $125^{\circ} \mathrm{C}$ for $30 \mathrm{~min}$. Sample solutions, in 5-50 $\mu \mathrm{g}$ concentration range, were prepared. For each bacterial strain, three test tubes were prepared i.e., S. aureus and E. coli. In above autoclaved tubes, $2 \mathrm{~mL}$ of LB Broth and $20 \mu \mathrm{L}$ of bacterial strain were added, followed by the addition of stocks of $5 \mu \mathrm{L}$, $10 \mu \mathrm{L}, 20 \mu \mathrm{L}$ containing $5.0 \mu \mathrm{g}, 12.5 \mu \mathrm{g}$ and $50 \mu \mathrm{g}$. Then these tubes were incubated at $37^{\circ} \mathrm{C}$ for $72 \mathrm{~h}$. At $600 \mathrm{~nm}$, OD of each medium and control medium was measured. A comparative study for synthesized compounds was made with Graph plotted between concentration and OD of compounds.

Well diffusion test [28-32] was performed to evaluate Antifungal activity of compounds using PDA (potato dextrose agar). Inoculum was prepared by using a $24 \mathrm{~h}$ yeast culture of PDA. Suspension was made by using sterile saline solution $(0.85 \%)$. Turbidity of above suspension was attuned by spectrophotometer at $600 \mathrm{~nm}$ for getting final concentration matching with $0.5 \mathrm{McFarland}$ standard. Then Agar medium was autoclaved for $30 \mathrm{~min}$ at $120^{\circ} \mathrm{C}$ and cooled at $50^{\circ} \mathrm{C}$ and inoculated with $1.0 \mathrm{~mL}$ of above suspension having absorbance 0.5 . Inoculated medium was then poured into all assay plates $9 \mathrm{~cm}$ in diameter and were allowed to cool down until solidified. Equidistance four wells $6 \mathrm{~mm}$ in diameter were cut out of agar upon solidification. $6 \mu \mathrm{L}$ of medium, containing synthesized compounds, was added into these wells. Incubation of plates was done at $27^{\circ} \mathrm{C}$ for $48 \mathrm{~h}$. For each compound, MIC values in $\mu \mathrm{g} / \mathrm{mL}$ and zone of inhibition in $\mathrm{mm}$ were calculated, comparing it with standard antifungal isoconazol (ISC) in concentration $1.0 \mu \mathrm{g} / \mathrm{mL}$ in each plate as positive control. Results are mentioned in the Table 1.

\section{Antioxidant activity}

In-vitro antioxidant activity of synthesized compounds, using DDPH, was evaluated by a reported method [33]. In order to produce precise results, all compounds were run in triplicate. Trolox was used for standard curve. Relative concentrations of compounds were determined using $\mathrm{R}^{2}$ value, and scavenging percentage that directly represents the antioxidant activity was calculated using formula i.e.,

Inhibition $\%=\left(1-\right.$ sample $_{530} /$ blank $\left._{530}\right) \times 100$

Results are given in Table 2.

\section{Results}

\section{Spectral characterization of sulfonamides}

$(6 R, 7 R)-3-($ acetoxymethyl)-7-((Z)-2-(methoxyimino)-2-(2-(4methylphenylsulfonamido)thiazol-4-yl)acetamido)-8-oxo-5-thia1-azabicyclo [4.2.0] oct-2-ene-2-carboxylic acid (HR1): Yield: $82 \%$, Colour: off white, m.p. (decomp.): $185^{\circ} \mathrm{C}$. FTIR $\left(\mathrm{cm}^{-1}\right): 1159.48(\mathrm{~S}=\mathrm{O}$ str), 1086.0 (C-N str), 1652.8 (C=O), 819.62 (C-S str), 958.9 (S-N str), $3368.2(-\mathrm{OH}) .{ }^{1} \mathrm{HNMR}$ (DMSO-d6, ppm): 11.08 (1H, s, -COOH), 8.09$8.12(1 \mathrm{H}, \mathrm{d}, \mathrm{J}=6.0,-\mathrm{NHCO}), 7.82-7.84(2 \mathrm{H}, \mathrm{d}, \mathrm{J}=8.2,-\mathrm{ArH}), 7.62(1 \mathrm{H}$, $\mathrm{s},-\mathrm{C}=\mathrm{CHS}-), 7.40-7.44(2 \mathrm{H}, \mathrm{d}, \mathrm{J}=8.3,-\mathrm{ArH}), 5.52-5.54(1 \mathrm{H}, \mathrm{d}, \mathrm{J}=6.1$, -NHCHCO-), 5.11-5.16 (1H, d, J=6.9, -SCHN-), $4.79\left(2 \mathrm{H}, \mathrm{s},-\mathrm{OCH}_{2}-\right)$, $4.02\left(1 \mathrm{H}, \mathrm{s},-\mathrm{NHSO}_{2}-\right), 3.92(3 \mathrm{H}, \mathrm{s},-\mathrm{OCH} 3), 3.19-3.23(1 \mathrm{H}, \mathrm{d}, \mathrm{J}=7.1$, -CHHS-), 3.08-3.10 (1H, d, J=7.0, -CHHS-), 2.35 (3H, s, - $\left.\mathrm{ArCH}_{3}\right), 2.27$ $\left(3 \mathrm{H}, \mathrm{s},-\mathrm{OCOCH}_{3}\right) \cdot{ }^{13} \mathrm{C}$ NMR $(\delta, \mathrm{ppm}):(170.27),(167.47),(164.52)$, (163.78), (161.73), (151.39), (144.97), (137.41), (136.82), (130.32), (129.38), (129.38), (129.38), (128.79), (128.79), (113.21), (63.27), (62.31), (60.69), (56.85), (24.23), (21.84), (20.92). MS (m/z, ESI): Calcd. for $\mathrm{C}_{23} \mathrm{H}_{23} \mathrm{~N}_{5} \mathrm{O}_{9} \mathrm{~S}_{3}{ }^{+}[\mathrm{M}+\mathrm{H}]^{+}$609.07; Found 609.05. Anal. Calcd. for $\mathrm{C}_{23} \mathrm{H}_{23} \mathrm{~N}_{5} \mathrm{O}_{9} \mathrm{~S}_{3}$ (609.05): C: $45.31 ; \mathrm{H}: 3.80 ; \mathrm{N}: 14.49$; Found: C: 45.13; H: 3.81; N: 14.45 .

1-cyclopropyl-6-fluoro-4-oxo-7-(4-tosylpiperazin-1-yl)-1,4dihydroquinoline-3-carboxylic acid (HR2): Yield: 75\%, Colour: Yellow, M.p. (decomp.): $240^{\circ} \mathrm{C}$. FTIR $\left(\mathrm{cm}^{-1}\right): 1155.2$ (S=O str), 1055.2 (C-N str), 1642.1 (C=O), 814.0 (C-S str), 932.5 (S-N str), 3388.3 (-OH). ${ }^{1} \mathrm{HNMR}$ (DMSO-d6, ppm): $11.08(1 \mathrm{H}, \mathrm{s},-\mathrm{COOH}), 8.68(1 \mathrm{H}$, $\mathrm{s},-\mathrm{CH}=\mathrm{CCOOH}), 8.15(1 \mathrm{H}, \mathrm{s},-\mathrm{ArH}), 7.78-7.81(2 \mathrm{H}, \mathrm{d}, \mathrm{J}=8.1,-\mathrm{ArH})$, 7.40-7.46 (2H, d, J=8.3, -ArH), $6.06(1 \mathrm{H}, \mathrm{s},-\mathrm{ArH}), 4.11-4.28(1 \mathrm{H}, \mathrm{q}$, $\mathrm{J}=7.2,-\mathrm{NCH}), 3.19-3.30\left(8 \mathrm{H}, \mathrm{d}, \mathrm{J}=6.2,-\mathrm{NCH}_{2} \mathrm{CH}_{2} \mathrm{~N}-\right), 2.36(3 \mathrm{H}, \mathrm{s}$, $\left.-\mathrm{ArCH}_{3}\right), 1.83-2.05\left(2 \mathrm{H}, \mathrm{dq}, \mathrm{J}=7.2,8.3-\mathrm{CHCHHCH}_{2}-\right), 1.11-1.37(2 \mathrm{H}$, $\left.\mathrm{dq}, \mathrm{J}=7.2,8.2-\mathrm{CHCHHCH}_{2}-\right) .{ }^{13} \mathrm{C} \mathrm{NMR}(\delta, \mathrm{ppm}):(176.67),(166.27)$, (152.87), (147.92), (146.38), (143.92), (137.48), (134.57), (129.42), (129.42), (128.26), (128.26), (115.87), (112.81), (109.79), (102.59), (53.37), (53.37), (48.51), (48.51), (35.85), (21.53), (7.59), (7.59). MS (m/z, ESI): Calcd. for $\mathrm{C}_{24} \mathrm{H}_{24} \mathrm{FN}_{3} \mathrm{O}_{5} \mathrm{~S}^{+}[\mathrm{M}+\mathrm{H}]^{+}$485.14; Found 485.04 . Anal. Calcd. for $\mathrm{C}_{24} \mathrm{H}_{24} \mathrm{FN}_{3} \mathrm{O}_{5} \mathrm{~S}$ (485.04): C: 59.37; $\mathrm{H}: 4.98$; N: 8.65; Found: C: $59.35 ; \mathrm{H}: 4.94 ; \mathrm{N}: 8.60$.

4-chloro-2-((furan-2-ylmethyl)amino)-5-(N-tosylsulfamoyl) benzoicacid (HR3): Yield: $62 \%$, Colour: White, M.p. (decomp.): $212^{\circ} \mathrm{C}$ FTIR $\left(\mathrm{cm}^{-1}\right): 1155.3$ ( $\mathrm{S}=\mathrm{O}$ str), 1050 (C-N str), $1640(\mathrm{C}=\mathrm{O}), 814$ (C-S 
Citation: Rehman H, Abdul Qadir M, Shad HA, Khan ZI (2017) One Pot Synthesis of Some Novel Sulfonamide Derivatives Containing -NH2 Group: Spectral Characterization and Biological Evaluation. Med Chem (Los Angeles) 7: 252-256. doi: 10.4172/2161-0444.1000465

\begin{tabular}{|c|c|c|c|c|c|c|c|c|}
\hline \multirow{3}{*}{ Compounds } & \multicolumn{4}{|c|}{ Bacterial Strains } & \multicolumn{4}{|c|}{ Fungal Strains } \\
\hline & \multicolumn{2}{|c|}{ Staphylococcus aureus, Gram (+) } & \multicolumn{2}{|c|}{ Escherichia coli, Gram (-) } & \multicolumn{2}{|c|}{ Aspergillum flavous } & \multicolumn{2}{|c|}{ Aspergillum niger } \\
\hline & MIC $^{b}$ & $\begin{array}{c}\text { Zone of } \\
\text { inhibition }^{\mathrm{a}}\end{array}$ & MIC $^{b}$ & $\begin{array}{l}\text { Zone of } \\
\text { inhibition }\end{array}$ & $\mathrm{MIC}^{\mathrm{b}}$ & $\begin{array}{c}\text { Zone of } \\
\text { inhibition }^{\mathrm{a}}\end{array}$ & MIC $^{b}$ & $\begin{array}{c}\text { Zone of } \\
\text { inhibition }\end{array}$ \\
\hline HR1 & $4.5 \times 10^{-3}$ & 2.8 & $4.9 \times 10^{-3}$ & 3 & $8.4 \times 10^{-3}$ & $10.4 \pm 0.22$ & $18.8 \times 10^{-3}$ & $9.6 \pm 0.10$ \\
\hline HR2 & $1.13 \times 10^{-3}$ & 7.5 & $1.54 \times 10^{-3}$ & 13 & $1.54 \times 10^{-3}$ & $24.5 \pm 0.32$ & $2.57 \times 10^{-3}$ & $14.3 \pm 0.23$ \\
\hline HR3 & $6.1 \times 10^{-3}$ & 3 & $9.2 \times 10^{-3}$ & 4.5 & $23.7 \times 10^{-3}$ & $14.9 \pm 0.11$ & $64.0 \times 10^{-3}$ & $13.9 \pm 0.22$ \\
\hline HR4 & $2.6 \times 10^{-3}$ & 1.5 & $4.9 \times 10^{-3}$ & 2.8 & $71.2 \times 10^{-3}$ & $25.2 \pm 0.12$ & $98.9 \times 10^{-3}$ & $17.1 \pm 0.12$ \\
\hline HR5 & $3.0 \times 10^{-3}$ & 1.6 & $4.2 \times 10^{-3}$ & 2.2 & $12.5 \times 10^{-3}$ & $23.5 \pm 0.10$ & $23.1 \times 10^{-3}$ & $17.1 \pm 0.11$ \\
\hline Standard* & $0.78 \times 10^{-3}$ & 0.2 & $0.15 \times 10^{-3}$ & 0.04 & $1.20 \times 10^{-3}$ & 30 & $1.82 \times 10^{-3}$ & 29.5 \\
\hline
\end{tabular}

a Zone of inhibition was measured in $\mathrm{mm}$; ${ }^{\mathrm{b}} \mathrm{MIC}$ (minimum inhibitory concentrations) were measured in mol/L; *Sulfmethoxazole is used as standard for bacterial strains and Isoconazole for fungal strains.

Table 1: Zone of inhibition ${ }^{\mathrm{a}}$ and $\mathrm{MIC}^{\mathrm{b}}$ of Sulfonamides against pathogenic bacterial and fungal strains.

\begin{tabular}{|c|c|c|}
\hline Compounds & \multicolumn{2}{|c}{ \% Antioxidant Activity } \\
\cline { 2 - 3 } & $\mathbf{4} \mathbf{~ m M}$ & $\mathbf{6} \mathbf{m M}$ \\
\hline HR1 & 1.22 \\
\hline HR2 & 1.76 \\
\hline HR4 & 1.21 & 13.60 \\
\hline HR5 & 1.19 & 15.72 \\
\hline Trolox & 1.18 & 12.06 \\
\hline
\end{tabular}

Table 2: Antioxidant Activity of Sulfonamide Derivatives HR1-HR5.

\begin{tabular}{|c|c|c|c|c|c|c|c|}
\hline Compounds & Molecular Formula & Molecular Weight & m.p. $\left({ }^{\circ} \mathrm{C}\right)$ & Time (h) & Yield (\%) & $\mathbf{R}_{\mathrm{f}}^{\mathrm{a}}$ & $\lambda_{\max }^{b}(n m)$ \\
\hline HR1 & $\mathrm{C}_{23} \mathrm{H}_{23} \mathrm{~N}_{5} \mathrm{O}_{9} \mathrm{~S}_{3}$ & 609.07 & 185 & 3 & 82 & 0.72 & 275 \\
\hline HR2 & $\mathrm{C}_{24} \mathrm{H}_{24} \mathrm{FN}_{3} \mathrm{O}_{5} \mathrm{~S}$ & 485.04 & 240 & 3 & 75 & 0.57 & 270 \\
\hline HR3 & $\mathrm{C}_{19} \mathrm{H}_{17} \mathrm{ClN}_{2} \mathrm{O}_{7} \mathrm{~S}_{2}$ & 484.05 & 212 & 3 & 62 & 0.53 & 280 \\
\hline HR4 & $\mathrm{C}_{27} \mathrm{H}_{31} \mathrm{CIN}_{2} \mathrm{O}_{7} \mathrm{~S}$ & 562.05 & 200 & 3 & 72 & 0.64 & 285 \\
\hline HR5 & $\mathrm{C}_{23} \mathrm{H}_{22} \mathrm{CIN}_{3} \mathrm{O}_{5} \mathrm{~S}_{2}$ & 519.09 & 187 & 3 & 75 & 0.68 & 280 \\
\hline
\end{tabular}

Table 3: Physiochemical and Analytical Data of Sulfonamides, (HR1-HR5).

str), 932 (S-N str), 3388 (-OH). ${ }^{1} \mathrm{HNMR}$ (DMSO-d6, ppm): $10.48(1 \mathrm{H}$, s, $-\mathrm{COOH}), 8.31(1 \mathrm{H}, \mathrm{s},-\mathrm{ArH}), 7.76-7.78(1 \mathrm{H}, \mathrm{d}, \mathrm{J}=7.2,-\mathrm{CH}=\mathrm{CH}-\mathrm{O}-)$, 7.66-7.68 $(2 \mathrm{H}, \mathrm{d}, \mathrm{J}=8.1,-\mathrm{ArH}), 7.39-7.42(2 \mathrm{H}, \mathrm{d}, \mathrm{J}=8.3,-\mathrm{ArH}), 7.25$ $(1 \mathrm{H}, \mathrm{s},-\mathrm{ArH}), 6.46-6.50(1 \mathrm{H}, \mathrm{t}, \mathrm{J}=6.9,-\mathrm{CH}=\mathrm{CH}-\mathrm{O}-), 6.25-6.27(1 \mathrm{H}, \mathrm{d}$, $\mathrm{J}=7.1,-\mathrm{CH}-\mathrm{CH}=\mathrm{CH}-\mathrm{O}-), 4.72-4.74\left(2 \mathrm{H}, \mathrm{d}, \mathrm{J}=6.3,-\mathrm{CH}_{2} \mathrm{NH}-\right), 4.01-4.04$ $\left(1 \mathrm{H}, \mathrm{t}, \mathrm{J}=6.1,-\mathrm{CH}_{2} \mathrm{NH}-\right), 2.37\left(3 \mathrm{H}, \mathrm{s},-\mathrm{ArCH}_{3}\right), 2.11\left(1 \mathrm{H}, \mathrm{s},-\mathrm{NHSO}_{2}-\right.$ ). ${ }^{13} \mathrm{CNMR}(\delta, \mathrm{ppm})$ : (169.92), (154.47), (142.17), (141.35), (137.74), (137.72), (136.21), (128.79), (128.79), (129.38), (128.71), (128.71), (125.57), (114.35), (110.79), (106.55), (105.26), (40.17), (21.42). MS (m/z, ESI): Calcd. for $\mathrm{C}_{19} \mathrm{H}_{17} \mathrm{ClN}_{2} \mathrm{O}_{7} \mathrm{~S}_{2}{ }^{+}[\mathrm{M}+\mathrm{H}]^{+}$484.02; Found 484.05. Anal. Calcd. for $\mathrm{C}_{19} \mathrm{H}_{17} \mathrm{CIN}_{2} \mathrm{O}_{7} \mathrm{~S}_{2}(484.05)$ : C: $47.09 ; \mathrm{H}: 3.41 ; \mathrm{Cl}: 7.31 ; \mathrm{N}$ : 5.78; S:13.22; Found: C: 47.01; H: 3.47; N: 5.76; Cl: 7.27; S:13.20.

3-ethyl-5-methyl-4-(2-chlorophenyl)-6-methyl-2-((2-(4methylphenylsulfonamido)ethoxy)methyl)-1,4-dihydropyridine3,5-dicarboxylate (HR4): Yield: 72\%, Colour: White, M.p. (decomp.): $200^{\circ} \mathrm{C}$. FTIR $\left(\mathrm{cm}^{-1}\right): 1150$ (S=O str), 1048 (C-N str), 1645 (C=O), 813 (C-S str), 930 (S-N str), 3380 (-OH). ${ }^{1} \mathrm{H}$ NMR (400 MHz, DMS-d6, $\delta$ ) 7.94-7.98 (2H, m, -ArH), 7.76-7.79 (1H, t, J=5.2, $\left.-\mathrm{NHSO}_{2}\right), 7.59-7.61$ $(1 \mathrm{H}, \mathrm{d}, \mathrm{J}=8.0,-\mathrm{ArH}), 7.40-7.43(2 \mathrm{H}, \mathrm{d}, \mathrm{J}=7.9,-\mathrm{ArH}), 7.14-7.20(3 \mathrm{H}, \mathrm{m}$, -ArH), $4.78(1 \mathrm{H}, \mathrm{s},-\mathrm{CHArCl}), 4.20-4.29\left(2 \mathrm{H}, \mathrm{q}, \mathrm{J}=6.2,-\mathrm{OCH}_{2} \mathrm{CH}_{3}\right)$, $4.07\left(2 \mathrm{H}, \mathrm{s},-\mathrm{CH}_{2} \mathrm{OCH}_{2} \mathrm{CH}_{2}-\right), 3.87\left(3 \mathrm{H}, \mathrm{s},-\mathrm{OCOCH}_{3}\right), 3.76-3.78(2 \mathrm{H}, \mathrm{t}$, $\left.\mathrm{J}=6.5,-\mathrm{OCH}_{2} \mathrm{CH}_{2} \mathrm{NH}-\right), 3.42-3.47\left(2 \mathrm{H}, \mathrm{q}, \mathrm{J}=6.2,-\mathrm{OCH}_{2} \mathrm{CH}_{2} \mathrm{NH}-\right), 2.36$ $\left(3 \mathrm{H}, \mathrm{s},-\mathrm{ArCH}_{3}\right), 2.24\left(3 \mathrm{H}, \mathrm{s},-\mathrm{CH}_{3}\right), 1.56(1 \mathrm{H}, \mathrm{s},-\mathrm{NH}-), 1.31-1.37(3 \mathrm{H}$, t, J=6.9, $\left.-\mathrm{OCH}_{2} \mathrm{CH}_{3}\right) .{ }^{13} \mathrm{CNMR}(\delta, \mathrm{ppm})$ : (167.37), (167.37), (149.47), (145.67), (143.59),(143.31), (137.59), (137.59), (131.58), (129.74), (129.74), (128.32), (128.32), (128.31), (126.63), (126.63), (126.21), (104.71), (100.73), (100.73), (70.89), (69.72), (61.97), (52.41), (21.53) (19.54), (14.32). MS (m/z, ESI): Calcd. for $\mathrm{C}_{27} \mathrm{H}_{31} \mathrm{ClN}_{2} \mathrm{O}_{7} \mathrm{~S}^{+}[\mathrm{M}+\mathrm{H}]^{+}$ 562.1; Found 562.05. Anal. Calcd. for $\mathrm{C}_{27} \mathrm{H}_{31} \mathrm{CIN}_{2} \mathrm{O}_{7} \mathrm{~S}$ (562.15): C:
57.59; H: 5.55; N: 4.98; O: 19.89; Cl: 6.30; S: 5.96; Found: C: 57.54; H: 5.53; N: 4.94; O: 19.87; Cl: 6.27; S: 5.67 .

4-chloro-N-(2-methylindolin-1-yl)-3-(N-tosylsulfamoyl) benzamide (HR5): Yield: $75 \%$, Colour: Yellow, M.p. (decomp.): $187^{\circ} \mathrm{C}$ FTIR $\left(\mathrm{cm}^{-1}\right): 1148$ (S=O str), 1055 (C-N str), $1640(\mathrm{C}=\mathrm{O}), 814$ (C-S str), 932 (S-N str), 3388 (-OH). ${ }^{1} \mathrm{H}$ NMR (DMSO-d6, ppm): 2.35 (s, $\left.6 \mathrm{H}, \mathrm{CH}_{3}\right), 6.9(\mathrm{~s}, 1 \mathrm{H}$, pyrimidine $), 7.2-7.5(\mathrm{~m}, 3 \mathrm{H}, \mathrm{Cl}-\mathrm{Ph}), 7.7-8.2(\mathrm{~m}$ $4 \mathrm{H}, \mathrm{N}-\mathrm{Ph}$ ), 8.91 (s, $1 \mathrm{H}$, azomethine), 8.9 (s, 1H, SO $\left.\mathrm{S}_{2} \mathrm{NH}-\right), 12.42$ (s, $1 \mathrm{H}, \mathrm{OH}) .{ }^{13} \mathrm{CNMR}(\delta, \mathrm{ppm})$ : (166.37), (160.15), (151.67), (139.68), (137.78), (136.41), (134.27), (133.37), (132.45), (129.71), (129.71), (129.42), (128.38), (128.38), (126.99), (126.09), (125.41), (122.53), (121.81), (108.23), (36.71), (21.84), (18.32). MS (m/z, ESI): Calcd. for $\mathrm{C}_{23} \mathrm{H}_{22} \mathrm{ClN}_{3} \mathrm{O}_{5} \mathrm{~S}_{2}{ }^{+}[\mathrm{M}+\mathrm{H}]^{+}$519.07; Found 519.09. Anal. Calcd. for $\mathrm{C}_{23} \mathrm{H}_{22} \mathrm{CIN}_{3} \mathrm{O}_{5} \mathrm{~S}_{2}(519.09$ ): C: $53.12 ; \mathrm{H}: 4.26 ; \mathrm{N}: 8.08 ; \mathrm{O}: 15.38 \mathrm{Cl}: 6.82$; S: 12.33 ; Found: C: $53.02 ; \mathrm{H}: 4.12$; N: 8.06; O: $15.37 \mathrm{Cl}: 6.80$; S: 12.31.

\section{Discussion}

A series of five sulfonamides were synthesized using efficient method based on Hinsberg Test and details of reaction conditions are explained in experimental section. The compounds HR1, HR2 and HR5 were obtained in excellent yield (above 75\%) while the HR3 and HR4 gave the good yield (above 62\%). Conformation of compounds was done by elemental analysis and measurement of absorption maximum $\left(\lambda_{\text {max }}\right)$ provided the justification. The physiochemical and analytical data of synthesized sulfonamides are presented in Table 3 . The synthesized compounds were characterized by FT-IR; the characteristics band at $1148-1155.5 \mathrm{~cm}^{-1}$ of $\mathrm{S}=\mathrm{O}$ stretching and $1048-1055 \mathrm{~cm}^{-1}$ for $(\mathrm{C}-\mathrm{N})$ and $813-814 \mathrm{~cm}^{-1}(\mathrm{C}-\mathrm{S})$ and $930-958.9 \mathrm{~cm}^{-1}(\mathrm{~S}-\mathrm{N})$ for all compounds reveals the formation of sulfonamides. In $\mathrm{HR} 1$ and $\mathrm{HR} 2$, peaks of $\mathrm{Ar}-\mathrm{CH}_{3}$ were 
Citation: Rehman H, Abdul Qadir M, Shad HA, Khan ZI (2017) One Pot Synthesis of Some Novel Sulfonamide Derivatives Containing -NH2 Group: Spectral Characterization and Biological Evaluation. Med Chem (Los Angeles) 7: 252-256. doi: 10.4172/2161-0444. 1000465

found in their concerned region i.e., 2.34-2.36 ppm. In HR1 there is a stereo-centre i.e., $-\mathrm{CH}_{2} \mathrm{H}_{b} \mathrm{~S}$-, both protons attached to carbon atom are in different environment. That's why they couple with each other to give their own doublets. A broad triplet was found in $\mathbf{H R 2}$ due to a six member ring containing two nitrogen atoms i.e., $-\mathrm{N}_{2}\left(\mathrm{CH}_{2}\right)_{4}$ - at 3.19$3.30 \mathrm{ppm}$. In $\mathbf{H R} 3$ and $\mathbf{H R 4},-\mathrm{ArCH}_{3}$ peaks were recorded at 2.37 and $2.36 \mathrm{ppm}$ respectively. In both compounds chemical shift values of - $\mathrm{ArH}$ which are in the vicinity of chlorine containing carbon were shifted to the downfield side due to electron withdrawing inductive effect of - $\mathrm{Cl}$ atom than other -ArH. In case of HR4 there is an ethyl group which is directly attached to the ester group. Methyl group $\left(-\mathrm{CH}_{3}\right)$ showed its triplet at 1.31-1.37 ppm and methylene group $\left(-\mathrm{CH}_{2}-\right)$ showed its quartet at 4.20-4.29 ppm. In HR5 there are two $-\mathrm{CH}_{3}$ groups, one group is aromatic methyl and second group is attached to a five member ring. Their peaks were found at $2.35 \mathrm{ppm}$ and $1.25-1.27 \mathrm{ppm}$ respectively. Similarly, chemical shift values of - $\mathrm{ArH}$ in - $\mathrm{Cl}$ containing ring has been shifted to down field side than other aromatic protons. This shifting is due to the high electronegativity of chlorine atom and its electron withdrawing effect. Developed compounds were also screened for their antioxidant and antimicrobial activities. All exhibited moderate activity but potential activity with $15.75 \%$ at $6 \mathrm{mM}$ was shown by compound HR2. Compounds HR4 and HR2 exhibited good activities against fungal strain A. flavous almost comparable with the reference Isoconazole. MIC values and zone of inhibition are presented in Table 1.

\section{Conclusion}

In conclusion, sulfonamides derivatives of five novel compounds, HR1-HR5, were synthesized and their antioxidant, antimicrobial and cytotoxicity test were also done. Remarkable antioxidant activity of concerned compounds guided and motivated us about their possible clinical significance. Antimicrobial activity was not so pronounced. Compound HR4 showed remarkable antifungal results, but compound HR2 was found to have potential antioxidant activity. However they did not give so pronounced cytotoxic effects.

\section{Acknowledgements}

Hajira Rehman gratefully acknowledges the cooperation of Director, Institute of Chemistry University of Punjab, Lahore, Pakistan for providing me lab facilities. I am also thankful to HEC Pakistan for providing financial support to carry out this project.

\section{Conflict of Interest}

Authors declare that there is no conflict of interest regarding the publication of this paper.

\section{References}

1. Brown GM (1971) The Biosynthesis of Pteridines. Advances in Enzymology and Related Areas of Molecular Biology 35: 35-77.

2. Massah AR, Adibi H, Khodarahmi R, Abiri R, Majnooni MB, et al. (2008) Synthesis, in vitro antibacterial and carbonic anhydrase II inhibitory activities of $\mathrm{N}$-acylsulfonamides using silica sulfuric acid as an efficient catalyst under both solvent-free and heterogeneous conditions. Bioorganic Medicinal Chemistry 16: $5465-5472$

3. Casini J, Antel F, Abbate A, Scozzafava S, David H, et al. (2003) Identification of 3, 4-Dihydroisoquinoline-2 $(1 H)$-sulfonamides as Potent Carbonic Anhydrase Inhibitors: Synthesis, Biological Evaluation, and Enzyme- Ligand X-ray Studies. Bioorganic Medicinal Chemistry Letters 13: 841-845.

4. Thiry A, Dogne JM, Supuran CT, Masereel B (2008) Current Pharmaceutical Design 14: 661-671.

5. Gitto R, Agnello S, Ferro S, De Luca L, Vullo D, et al. (2010) Journal of Medicinal Chemistry 53: 2401-2408.

6. Supuran CT, Casini A, Scozzafava A (2003) Protease inhibitors of the sulfonamide type: anticancer, antiinflammatory, and antiviral agents. Medicinal Research Reviews 23: 535-558.
7. Cheng XC, Wang Q, Fang $H$, Xu WF (2008) Role of sulfonamide group in matrix metalloproteinase inhibitors. Current Medicinal Chemistry 15: 368-373.

8. Kuang R, Epp JB, Ruan S, Yu H, Huang P, et al. (1999) Therapeutic potentia of sulfamides as enzyme inhibitors. Journal of the American Chemical Society 121: 8128-8129.

9. Groutas WC, He S, Kuang R, Ruan S, Tu J, et al. (2001) Bioorganic Medicinal Chemistry 9: 1543-1548.

10. Zhong J, Gan X, Alliston KR, Lai Z, Yu H, et al. (2004) J Comb Chem 6: 556-563.

11. Winum JY, Scozzafava A, Montero JL, Supuran CT (2006) Medicinal Research Reviews 26: 767-792.

12. Maren TH (1967) Carbonic anhydrase: Chemistry, physiology, and inhibition. Physiological Reviews 47: 595-781.

13. Supuran CT, Innocenti A, Mastrolorenzo A, Scozzafava A (2004) Antiviral sulfonamide derivatives. Mini-Reviews in Medicinal Chemistry 4: 189-200.

14. Markgrem PO, Schaal W, Hamalainem M, Karlen A, Hallberg A, et al. (2002) Relationships between structure and interaction kinetics for HIV-1 protease inhibitors. Journal of Medicinal Chemistry 2: 5430-5439.

15. Stranix BR, Sauvé G, Bouzide A, Coté A, Sévigny JY, et al. (2004) Lysine sulfonamides as novel HIV-protease inhibitors: $N$-epsilon -distributed ureas. Bioorganic Medicinal Chemistry Letters 14: 3971-3974

16. Crespo R, De Bravo MG, Colinas PA, Bravo RD (2010) In vitro antitumor activity of $\mathrm{N}$-glycosyl sulfonamides. Bioorganic Medicinal Chemistry Letters 20 6469-6474.

17. Brown SD, Traczewski MM (2005) Synthesis and antibacterial activity of sulfonamides. SAR and DFT Studies. Journal of Antimicrobial Chemotherapy 55: $944-949$

18. Supuran CT, Scozzafava A (2002) Applications of carbonic anhydride inhibitors and activators in therapy. Expert Opinion on Therapeutic Patents 12: 217-242.

19. Supuran CT, Scozzafava A (2001) Carbonic Anhydrase Inhibitors. Current Medicinal Chemistry - Immunology Endocrine \& Metabolic 1: 61-97.

20. Supuran CT, Scozzafava A (2000) Carbonic anhydrase inhibitors and their therapeutic potential. Expert Opinion on Therapeutic Patents 10: 575.

21. Das B, Reddy VS, Reddy MR (2004) An efficient and selective tosylation of alcohols with p-toluenesulfonic acid. Tetrahedron Letters 45: 6717-6719.

22. Chohan ZH, Hazoor A. Shad S (2008) Structural elucidation and biological significance of 2-hydroxy-1-naphthaldehyde derived sulfonamides and their first row d-transition metal chelates. Journal of Enzyme Inhibition and Medicinal Chemistry 23: 369-379.

23. Chohan ZH, Hazoor AS, Faiz-ul-Hassan N (2009) Synthesis, characterization and biological properties of sulfonamide-derived compounds and their transition metal complexes. Applied Organometallic Chemistry 23: 319-328.

24. Chohan ZH, Hazoor AS, Moulay H, Youssoufi Y, Hadda TB (2010) Some new biologically active metal-based Sulfonamide. European Journal of Medicinal Chemistry 45: 2893-2901.

25. Chohan ZH, Hazoor AS (2011) Sulfonamide-derived compounds and thei transition metal complexes: synthesis, biological evaluation and $\mathrm{X}$-ray structure of 4-bromo-2-[(E)-\{4-[(3,4-dimethylisoxazol-5-yl)sulfamoyl]phenyl $\}$ iminiomethyl] phenolate. Applied Organometallic Chemistry 25: 591-600.

26. Caddick S, Wilden JD, Judd DB (2004) Direct synthesis of sulfonamides and activated sulfonate esters from sulfonic acids. Journal of Organic Chemistry 126: 1024-1042.

27. Sezonov G, Joseleau-Petit D, Dari R (2007) Escherichia coli physiology in Luria-Bertani broth. Journal of Bacteriology 189: 8746-8749.

28. Magalia S, Camero T (1997) Susceptibilidad de Candida albicans 'invitro' mediante los pozos de difusión. Bolet'ınVenezolanode Infectolog'ıa 7: 5-8.

29. Magaldi S, Camero T (1998) Pruebas de sensibilidadde Candida albicansfrente a los de usocomercial. Bolet'ınSociedad Venezolana de Microbiologia 18: 16-20.

30. Magaldi S, Mata S, Camero T (1999) Determinacion de lasensibilidadantifúngica en agentes de cromomicosismediantelatecnica de los pozos de difusion. Antibioticos e Infeccion 7: 17-20.

31. Magaldi S, Mata S, Hartung C (2000) In-vitro susceptibility of 137 Candida sp. isolates from HIV positive patients to several antifungal drugs. Mycopathologia 149: 63-68. 
Citation: Rehman H, Abdul Qadir M, Shad HA, Khan Zl (2017) One Pot Synthesis of Some Novel Sulfonamide Derivatives Containing -NH2 Group: Spectral Characterization and Biological Evaluation. Med Chem (Los Angeles) 7: 252-256. doi: 10.4172/2161-0444.1000465

32. Magaldi S, Mata S, Hartung C (2001) In vitro susceptibility to fluconazole of Candida spp. isolates comparing three different methods. J Mycol Med 1: 123-126.
33. Mohammed $\mathrm{H}$ (2009) Natural and synthetic flavonoid derivatives with potential antioxidant and anticancer activities. 